\title{
Post-traumatic Stress Disorder and Mental Health Comorbidities: Results from the São Paulo Megacity Mental Health Survey
}

Aline Cristina de Oliveira Costa', Maria Carmen Viana ${ }^{2}$, Evandro da Silva Freire Coutinho'.

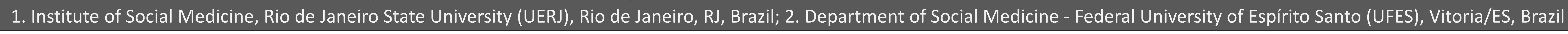

\section{Aims}

To estimate the prevalence of posttraumatic stress disorder (PTSD) mental health comorbidities and to describe the temporal sequence of those diagnoses in adult residents of the São Paulo Metropolitan Area (SPMA), Brazil.

\section{Background}

Comorbidity of PTSD with other psychiatric disorders has been consistently reported among war veterans, clinical samples and general population individuals. Such condition is predictive of worse clinical prognosis, reduced treatment response and higher impact on life quality when compared to PTSD alone.

\section{Study Overview}

The São Paulo

Megacity Mental

Health Survey is a

cross-sectional

population-based

study that

integrates the

World Mental

Health Survey

Initiative.

The diagnostic instrument

applied was World

Mental Health

Survey Version of

the Composite

International

Diagnostic

Interview, a fully

structured

diagnostic

interview.

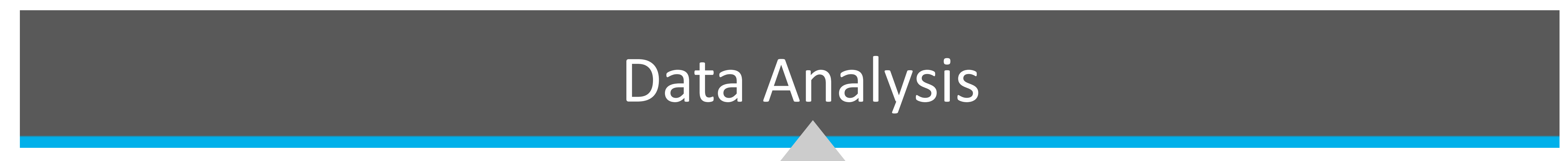

The prevalence of each comorbid disorder in the samples with and without PTSD and its respective $95 \%$ confidence interval was estimated.

The temporal sequence of comorbid diagnosis was assessed through the reported age of onset of mental disorders symptomatology.
Based on the time lag between the age of onset of the comorbid disorder and PTSD, the comorbid disorder was classified as preceding PTSD, occurring after PTSD onset, or as with

concomitant onset, when both stated at the same year.
Analyses were conducted using STATA, version 12 (StataCorp, 2011) and were carried out using svy routine, which adjusts population estimates for complex sampling designs.

\section{Results}

Table 1 - Prevalence of mental disorders in individuals with PTSD and without PTSD. São Paulo Megacity Mental Health Survey (N=2,942).

\begin{tabular}{|lccc|}
\hline & With PTSD & Without PTSD & Prevalence Ratio \\
\hline Anxiety Disorders & $\%(95 \% \mathrm{Cl})$ & $\%(95 \% \mathrm{Cl})$ & $\mathrm{PR}(95 \% \mathrm{Cl})$ \\
Mood Disorders & $65.2(54.05-74.9)$ & $25.0(23.08-27.03)$ & $2.6(2.2 ; 3.1)$ \\
Substance Use Disorders & $66.1(53.3-76.9)$ & $18.5(16.4-20.8)$ & $3.6(2.9 ; 4.3)$ \\
\hline Any Mental Disorder & $28.9(18.6,42.0)$ & $11.2(9.8,12.7)$ & $2.6(1.8 ; 3.8)$ \\
\hline
\end{tabular}

Table 2 - Temporal sequence of PTSD and comorbid mental disorders ( $N=2,942)$.

sample of adult residents of the SPMA was assessed for PTSD and other mental health disorders.

The response rate was $81.3 \%$. The study was approved by the Ethical and Research Committee, School of Medicine/São Paulo University.

\begin{tabular}{|lcccccc|} 
Anxiety Disorders & 75.8 & $(59.5-92.2)$ & 5.1 & $(0-10.2)$ & 19.1 & $(5.5-32.6)$ \\
\hline Mood Disorders & 38.5 & $(26.5-50.5)$ & 21.9 & $(14.1-29.6)$ & 39.6 & $(25.5-53.7)$ \\
\hline Substance Use Disorders & 48.9 & $(8.5-59.3)$ & 6.1 & $(0.0-23.0)$ & 45.0 & $(10.0-80.0)$
\end{tabular}

\section{Comments}

This study found high prevalence of mental disorders in comorbidity with PTSD in the general population of SPMA, Brazil. A better understanding of temporal patterns of onset of the comorbid condition may contribute to the risk assessment of its development. Our results reinforce the importance of considering comorbidity in PTSD clinical management and academic research.

Comorbidity posterior to PTSD

$$
\begin{gathered}
95 \% \text { IC } \\
(5.5-32.6) \\
(25.5-53.7) \\
(10.0-80.0)
\end{gathered}
$$

